\title{
In-plane anisotropy of the electronic structure for the charge/orbital-ordered state in half-doped manganite with layered structure
}

\author{
Y. S. Lee, ${ }^{1,2}$ S. Onoda, ${ }^{1}$ T. Arima,${ }^{1,3}$ Y. Tokunaga,,${ }^{1}$ J. P. He,${ }^{1}$ Y. Kaneko, ${ }^{1}$ N. Nagaosa,${ }^{4,5}$ and Y. Tokura ${ }^{1,4,5}$ \\ ${ }^{1}$ Spin Superstructure Project, ERATO, Japan Science and Technology Agency, Tsukuba 305-8562, Japan \\ ${ }^{2}$ Department of Physics, Soongsil University, Seoul 156-743, Korea \\ ${ }^{3}$ Institute of Multidisciplinary Research for Advanced Materials, Tohoku University, Sendai 980-8577, Japan \\ ${ }^{4}$ Correlated Electron Research Center, National Institute of Advanced Industrial Science and Technology, \\ AIST Tsukuba central 4, Tsukuba 305-8562, Japan \\ ${ }^{5}$ Department of Applied Physics, University of Tokyo, Tokyo 113-8656, Japan
}

(Dated: October 31, 2018)

\begin{abstract}
We report on the in-plane anisotropy of the electronic response in the spin/charge/orbital ordered phase of a half-doped layered-structure manganite. The optical conductivity spectra for a single domain of $\mathrm{Eu}_{1 / 2} \mathrm{Ca}_{3 / 2} \mathrm{MnO}_{4}$ unambiguously show the anisotropic charge dynamics which well corresponds to the theoretical calculation: the optical conductivity with the polarization along the zigzag ferromagnetic chain direction exhibits a smaller gap and a larger intensity at lower energies than that of the perpendicular polarization mostly due to the charge/orbital ordering and the associated quantum interference effect.
\end{abstract}

PACS numbers: 75.47.Gk, 75.30.Et, 78.40.Ha

Recent studies on strongly correlated electronic systems have revealed many unexpected phenomena due to interplay among the spin, charge and orbital degrees of freedom. In particular, nano-scale self-organization and the associated complexity of electronic systems are issues of current interest. One of the representative phenomena is the spin/charge/orbital ordering in transition metal oxides (TMOs), which governs the electrical, optical, and mechanical properties of the system [1]. Doping carriers into the parent Mott insulating compound causes a variety of ordering phenomena including one-dimensional (1D) stripe formation as observed in some layered $\mathrm{Cu}-$ and Ni-oxides. In Mn-oxides near half-doping there appears a cooperatively ordered pattern of charge, spin, and orbital, referred to as CE-type magnetic order coupled to the charge/orbital ordering (CO-OO) 2, 3, [4, 5, 6], which is involved in the metal-insulator transition with magnetic field and other various control parameters [7]. Below the $\mathrm{CO}-\mathrm{OO}$ transition temperature $T_{\mathrm{CO}-\mathrm{OO}}$, charges are ordered in a checkerboard-type with ideally alternating $\mathrm{Mn}^{3+} / \mathrm{Mn}^{4+}$ sites, i.e., the $3 d e_{g}^{1} / e_{g}^{0}$ configuration in addition to localized $t_{2 g}$ spins, and $e_{g}$ orbitals are aligned in the zigzag chain type. The direction of $e_{g}$ orbitals defines the zigzag chain and stripe [Fig. 1(c)]. Below the Néel temperature $T_{N}$, which is usually far below $T_{C O-O O}$, the spins of $3 d$ electrons at $\mathrm{Mn}$ sites are ordered ferromagnetically along the orbital zigzag chain path, while antiferromagnetically between the chains. This well-organized charge/spin/orbital ordering occurs in the $a b$ plane for a variety of manganites including layered compounds, and generically has the effective twodimensional nature in electronic response even for the pseudo-cubic perovskite [8].

To access the attribute of the charge/orbital ordering in more depth, we pay attention to the in-plane anisotropy of the electronic structure in the CO-OO phase, in particular, the optical response. The spin order may invoke the simplest trivial source of the anisotropy. Namely, well below $T_{N}$ where the ferromagnetic chains are coupled antiferromagnetically, the local electron hopping between the adjacent chains costs a larger energy typically of the order of $J_{H} S\left(J_{H}\right.$ :Hund's rule coupling, $S$ : magnitude of the $t_{2 g}$ spin), than that along the zigzag chain path. Since the optical processes occur locally, they are insensitive to the range of the magnetic ordering, and the anisotropy remains even above $T_{N}$. This scenario, however, is oversimplified and may not apply directly to real experimental systems that show the drastic change of electronic response below $T_{C O-O O}$, which is even well above $T_{N}$. Therefore, we must seriously consider the effects of CO-OO on the anisotropy of the low-energy optical response.

To elucidate the effects of CO-OO in the in-plane optical response, we take the following minimal model for a ferromagnetic zigzag chain in the CE phase;

$$
\begin{aligned}
\mathcal{H}= & -\sum_{\mathbf{r}, \mathbf{r}^{\prime}} \sum_{\alpha, \beta=1,2} t_{\mathbf{r}, \mathbf{r}^{\prime}}^{\alpha, \beta} c_{\mathbf{r} \alpha}^{\dagger} c_{\mathbf{r}^{\prime} \beta}+U \sum_{\mathbf{r}} n_{\mathbf{r} 1} n_{\mathbf{r} 2} \\
& +E_{J T} \sum_{\mathbf{r}}\left(u_{\mathbf{r}}^{2}+v_{\mathbf{r}}^{2}-2 u_{\mathbf{r}}\left(n_{\mathbf{r} 1}-n_{\mathbf{r} 2}\right)-2 v_{\mathbf{r}}\left(c_{\mathbf{r} 1}^{\dagger} c_{\mathbf{r} 2}+c_{\mathbf{r} 2}^{\dagger} c_{\mathbf{r} 1}\right)()\right)
\end{aligned}
$$

With the annihilation (creation) operators $c_{\mathbf{r} \alpha}\left(c_{\mathbf{r} \alpha}^{\dagger}\right)$ and the number operator $n_{\mathbf{r} \alpha}=c_{\mathbf{r} \alpha}^{\dagger} c_{\mathbf{r} \alpha}$ of the electron at site $\mathbf{r}$ with the $e_{g}$ orbital $\alpha\left(=1\right.$ or 2 for $d_{3 z^{2}-r^{2}}$ or $d_{x^{2}-y^{2}}$ respectively), the Hamiltonian given by Eq. (1) contains the orbital-dependent electron transfer $t_{\mathbf{r}, \mathbf{r}}^{\alpha, \beta}$ between the adjacent sites $\mathbf{r}$ and $\mathbf{r}^{\prime}$ with the same spin polarization as the first term, and the local Coulomb and the JahnTeller interactions $U$ and $E_{J T}$ as the second and the third terms, respectively. $u_{\mathbf{r}}$ and $v_{\mathbf{r}}$ denote the local Jahn-Teller modes for the $\mathrm{MnO}_{6}$ octahedron centered at the Mn site $\mathbf{r}$, as shown in the inset of Fig. 1 (b). We have neglected the phonon dynamics of these Jahn-Teller modes. This frozen Jahn-Teller distortion corresponds 


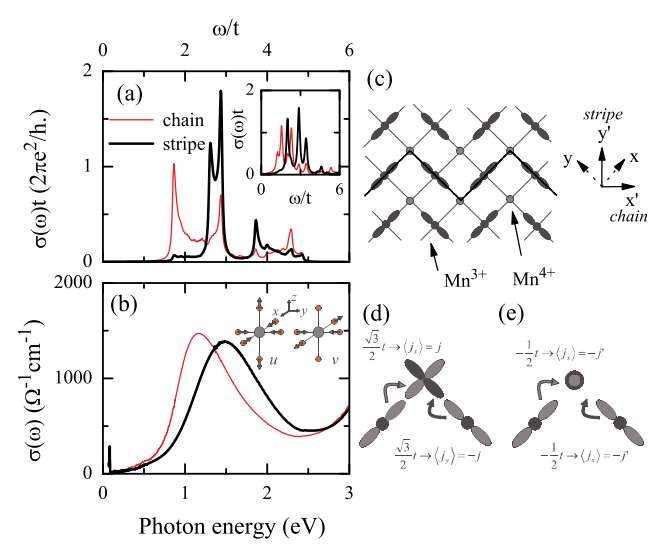

FIG. 1: (Color online) (a) Optical conductivity spectra along the chain and the stripe directions from (a) the calculation using the Hartree-Fock method and (b) the experiment for the single domain of ECMO. In the inset of (a) the result of the exact diagonalization study is displayed. In the inset of (b) two Jahn-Teller modes, $u$ and $v$, are drawn with a $\mathrm{MnO}_{6}$ octahedron. (c) Schematic diagram for the charge/orbital ordered state at half-doping. Right bottom panels depict difference in the phases for the local hopping and current matrix elements from the $d_{3 x^{2}-r^{2}} / d_{3 y^{2}-r^{2}}$ orbitals at the electron-rich sites to (d) the $d_{x^{2}-y^{2}}$ and (e) the $d_{3 z^{2}-r^{2}}$ orbitals at the electronpoor ones.

to the Frank-Condon approximation which is justified for the strong electron-phonon coupling case as in the present situation. As for the orbital and directional dependencies of the transfer integrals, we take the SlaterKoster's value [9] with $V_{d d \sigma}=t=0.25 \mathrm{eV}$, which is taken as the energy unit.

We solve this Hamiltonian within the Hartree-Fock approximation to obtain the self-consistent values for the expectation values $\left\langle c_{\mathbf{r} \alpha}^{\dagger} c_{\mathbf{r}^{\prime} \alpha^{\prime}}\right\rangle$ as well as the Jahn-Teller distortions $u_{\mathbf{r}}$ and $v_{\mathbf{r}}$. We note that Hartree-Fock treatment provides a reasonable description of the ground state even at the large $U$. Since the result is insensitive to the value of $U$, we may take a typical value for transitionmetal ion, $U=8 t$. In the present case of half doping, we have obtained the $\mathrm{CO}-\mathrm{OO}$ pattern characterized by the charge disproportionation $\delta n$ and the dominant orbital character of $d_{3 x^{2}-r^{2}} / d_{3 y^{2}-r^{2}}$ at the electron-rich $\left(\frac{1+\delta n}{2}\right)$ sites and $d_{x^{2}-y^{2}}$ at the electron-poor $\left(\frac{1-\delta n}{2}\right)$ ones. For this ordering the Jahn-Teller coupling is indispensable, as previously pointed out [10], and the ground-state properties are insensitive to the choice of $U$. Then, we employ the value $E_{J T}=2 t$ to reproduce the experimental result of the optical gap. This choice of $E_{J T}=2 t$ gives the charge disproportionation of $\delta n=0.56$, which is intermediate between the uniform value $\delta n=0$ for $E_{J T}=0$ and the ideal value $\delta n=1$ for $E_{J T} \rightarrow \infty$.

Using the Hartree-Fock solution, we have calculated the optical conductivity $\sigma^{\text {chain }}$ and $\sigma^{\text {stripe }}$ along the chain and the stripe directions, respectively, with the corre- sponding current operators $J^{\text {chain }}=\left(J^{x}+J^{y}\right) / \sqrt{2}$ and $J^{\text {stripe }}=\left(J^{x}-J^{y}\right) \sqrt{2}$ where

$$
J^{x, y}=-\frac{1}{N} \sum_{\mathbf{k}, \alpha, \beta} \frac{\partial t_{\mathbf{k}}^{\alpha, \beta}}{\partial k_{x, y}} c_{\mathbf{k}, \alpha}^{\dagger} c_{\mathbf{k}, \beta}
$$

with $t_{\mathbf{k}}^{\alpha, \beta}=\sum_{\boldsymbol{\delta}} t_{\mathbf{r}, \mathbf{r}+\boldsymbol{\delta}}^{\alpha, \beta} e^{-i \mathbf{k} \cdot \boldsymbol{\delta}}$. The calculated results for $\sigma^{\text {chain }}$ and $\sigma^{\text {stripe }}$ in the case of $E_{J T}=2 t$ are shown in Fig. 1(a). This calculation reveals that the optical conductivity along the chain direction exhibits a smaller optical gap and a larger spectral weight distribution at lower energies than along the stripe direction. We have also done the exact-diagonalization study of the same model with the Jahn-Teller distortion being fixed at the ground state value (Frank-Condon approximation), and found that the global structure of $\sigma^{\text {chain }}$ and $\sigma^{\text {stripe }}$ and the emergence of the anisotropy do not alter even beyond the Hartree-Fock, as shown in the inset of Fig 1(a). The anisotropy originates from the difference in the phase factors for the combination of $J^{x}$ and $J^{y}$ in the orbitalordered state and is an increasing function of the COOO order parameters. More explicitly, the local hopping process from the $d_{3 x^{2}-r^{2}} / d_{3 y^{2}-r^{2}}$ orbitals at the electronrich sites to the $d_{x^{2}-y^{2}}$ orbital at the electron-poor site, which is shown in Fig. 1(d), exhibits additive and negative interference in the chain and the stripe directions, respectively, leading to the strong anisotropy, while the hopping to the $d_{3 z^{2}-r^{2}}$ orbital shown in Fig. 1(e) does not.

To address the in-plane anisotropy experimentally, the measurement for an in-plane twin-free single domain sample is highly required, but has not been performed so far due to a great difficulty to obtain a sample large enough for optical measurement $(\sim 100 \mu \mathrm{m}$ in case of using microscope probe). To overcome this technical problem we chose a 2D single layered structure which is quite suitable for probing the $\mathrm{Mn}-\mathrm{O}$ plane where the CO-OO occurs. Due to the two-dimensional electronic nature of the charge/orbital ordering concerned here, our study with layered manganites is directly applicable to the case of the pseudo-cubic perovskite. For the wellstudied half-doped material $\mathrm{La}_{1 / 2} \mathrm{Sr}_{3 / 2} \mathrm{MnO}_{4}$ (LSMO), a typical domain size was found to be $\sim 10 \times 10 \mu \mathrm{m}^{2}$ [11], not sufficiently large for our purpose. Considering that the lattice distortion by the small $A$-site ions helps the domain size larger we newly synthesized the $\mathrm{Eu}_{1 / 2} \mathrm{Ca}_{3 / 2} \mathrm{MnO}_{4}$ (ECMO) compound where the average size of $A$-site ions $(\sim 1.165 \AA)$ is much smaller than that in LSMO $(\sim 1.285 \AA)$ [12]. The $T_{C O-O O}$ for the ECMO compound is as high as $\sim 325 \mathrm{~K}$ perhaps due to the narrower bandwidth, which is identified from anomalies in resistivity and magnetization, and a superlattice peak in the electron diffraction pattern (EDP). The $T_{N}$ is estimated to be $120-130 \mathrm{~K}$ far below $T_{C O-O O}$ [13]. Using the polarized microscope we could identify the stripetype domain pattern in the cleaved $a b$ surface. While the typical widths of domains are $10-50 \mu \mathrm{m}$, we succeeded in finding an exceptionally large single domain with $\sim 300$ 


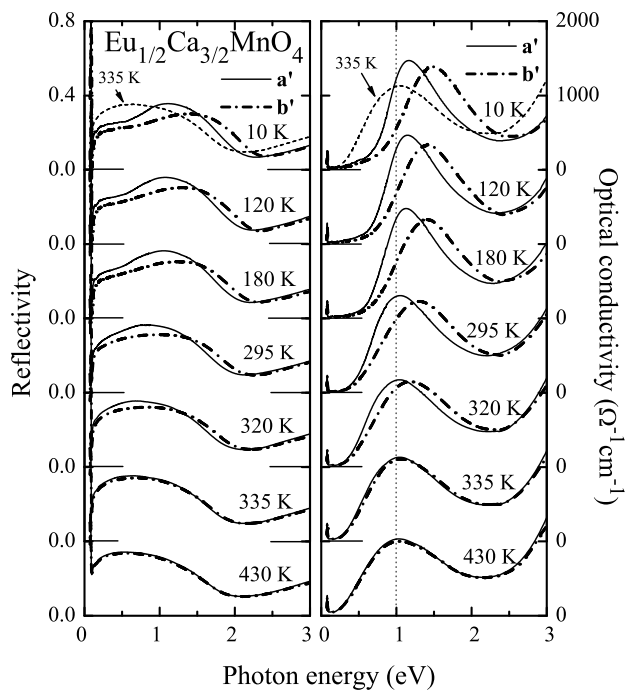

FIG. 2: Reflectivity spectra (left panels) and optical conductivity spectra (right panels) of $\mathrm{Eu}_{1 / 2} \mathrm{Ca}_{3 / 2} \mathrm{MnO}_{4}$ along the $a^{\prime}$ and $b^{\prime}$ axes. The panels at each temperature are shifted up in sequence. The $335 \mathrm{~K}$ data are included in the top panels to clarify the temperature-dependence. The dotted line in the right panels is for guidance.

$\mu \mathrm{m}$ width, which enabled us to perform the polarizationdependent optical measurement for the CO-OO phase.

$\mathrm{A} \mathrm{Eu}_{1 / 2} \mathrm{Ca}_{3 / 2} \mathrm{MnO}_{4}$ single crystal with half-doping was grown by the floating zone method. For the reflectivity measurement of a single domain, we used the microscopeequipped spectrometers with the beam size, $\sim 150 \times 100$ $\mu \mathrm{m}^{2}$. With proper polarizers the probed light is polarized along the optical axis $\left(a^{\prime}\right.$ or $\left.b^{\prime}\right)$, which is rotated by 45 degree from the crystal axes of the tetragonal cell. The EDP experiment on this material has revealed that the $a^{\prime}$ $\left(b^{\prime}\right)$ axis chosen for our measurement should correspond to the chain (stripe) direction. $T$-dependent reflectivity spectra $R(\omega)$ at nearly normal incidence were measured in a photon energy region from 0.06 to $5 \mathrm{eV}$ with the temperature variation from 10 to $430 \mathrm{~K}$. Since the anisotropic feature in $R(\omega)$ is terminated in the charge transfer excitation region above $2.5 \mathrm{eV}, T$-dependent $R(\omega)$ below $5 \mathrm{eV}$ are connected smoothly to room temperature $R(\omega)$ above 5 to $40 \mathrm{eV}$ obtained with a twinned sample. Optical conductivity spectra $\sigma(\omega)$ were obtained from the measured $R(\omega)$ using the Kramers-Kronig transformation.

Figure 2 shows the reflectivity and the optical conductivity spectra with polarization dependence at various $T$. Both of optical spectra along $a^{\prime}$ and $b^{\prime}$ axes show a similar trend with $T$, exhibiting significant changes related to the CO-OO. As $T$ is lowered below $T_{\mathrm{CO}-\mathrm{OO}}$, the reflectivity level is suppressed in a low energy region below $\sim 1 \mathrm{eV}$, while enhanced in the higher energy region. The $\sigma(\omega)$ calculated from the measured $R(\omega)$ exhibits that a distinct absorption below $2 \mathrm{eV}$, which is

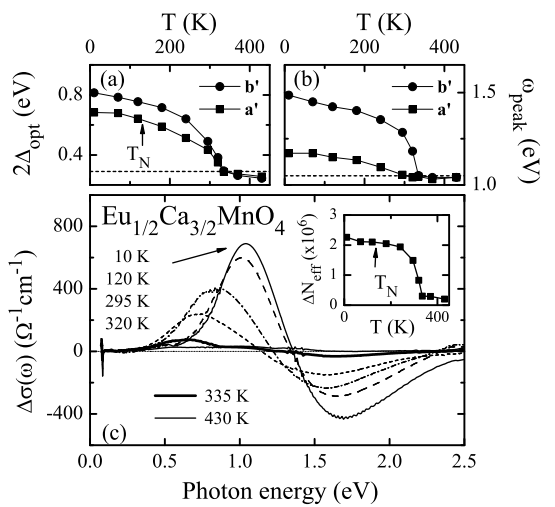

FIG. 3: (a) Optical gap, $2 \Delta_{\text {opt }}$ and (b) absorption peak below $2 \mathrm{eV}, \omega_{\text {peak }}$ along the $a^{\prime}$ and $b^{\prime}$ axes. (c) Differential conductivity $\Delta \sigma(\omega)=\sigma_{a^{\prime}}(\omega)-\sigma_{b^{\prime}}(\omega)$ at various temperatures. Inset displays the temperature dependence of $\Delta N_{\text {eff }}(\omega=1.3 \mathrm{eV})$. The unit is $\Omega^{-1} \mathrm{~cm}^{-2}$.

associated with the electron hopping from $\mathrm{Mn}^{3+}$ (occupied $e_{g}$ ) to $\mathrm{Mn}^{4+}$ (unoccupied $e_{g}$ ) 14], shifts to a higher energy with the sharper spectral shape at lower $T$ below $T_{C O-O O}$, leading to a larger optical gap. The overall spectral feature is in accord with the previous results for twinned LSMO samples 11, 15], and the related theoretical calculations [16, 17]. Apart from this common $T$-dependence, we could discern a clear development of anisotropy in optical spectra below $T_{C O-O O}$. The reflectivity spectra at $335 \mathrm{~K}$ (above $T_{C O-O O}$ ) appear to be nearly identical for both polarizations. In contrast, at $320 \mathrm{~K}$ (below $T_{\mathrm{CO}-\mathrm{OO}}$ ) the distinct anisotropy is identified; the level of reflectivity in the $b^{\prime}$ axis is lower below $1.3 \mathrm{eV}$, while higher above $1.3 \mathrm{eV}$, than in the $a^{\prime}$-axis. The difference between $R(\omega)$ along the two axes is larger at lower $T$. Similarly, the $\sigma(\omega)$ spectra polarized along the $b^{\prime}$-axis are distinguished from those along the $a^{\prime}$-axis below $T_{C O-O O}$, exhibiting the higher energy of absorption band and the larger optical gap.

To clarify the in-plane anisotropy, we display the polarization dependence of the optical gap, $2 \Delta_{\text {opt }}$, and the absorption position below $2 \mathrm{eV}, \omega_{\text {peak }}$, in Figs. 3(a) and $3(\mathrm{~b})$. The $2 \Delta_{\mathrm{opt}}$ values were estimated from crossing points of abscissa with linear extrapolations of $\sigma(\omega)$. At $T<T_{C O-O O}$ both the quantities have larger values along the $b^{\prime}$-axis than the $a^{\prime}$-axis. The anisotropic difference in $2 \Delta_{\text {opt }}$ and $\omega_{\text {peak }}$ are estimated to be $\sim 0.12 \mathrm{eV}$ and $\sim 0.35 \mathrm{eV}$, respectively, at the lowest temperature, and hence the degree of anisotropy is as large as $20-30 \%$. It is noted that such sizable anisotropy cannot originate from the orthorhombic crystal structure alone. While the phonon spectra reveal the lowering of crystal symmetry below $T_{C O-O O}$ [11, 18], the degree of orthorhombicity is too small $\left(b^{\prime} / a^{\prime}-1<0.01\right)$ to give rise to the significant electronic anisotropy observed here. In fact, the observed anisotropy above $T_{C O-O O}$, which is supposed 
to come from the inherent lattice orthorhombicity apart from CO-OO, is extremely small.

Notably, the electronic anisotropy unveiled by the experiments is in good agreement with the theoretical prediction as shown in Fig. 1. Because the $a^{\prime}\left(b^{\prime}\right)$ axis corresponds to the chain (stripe) direction, our combined experimental and theoretical studies unambiguously reveal the formation of the smaller energy gap state along the chain direction. This finding could be a strong evidence of more effective electron hopping along the chain direction in the CO-OO phase. Clearly the in-plane anisotropy identified below $T_{C O-O O}$ is attributed to the unidirectional zigzag chain arrangement of the $e_{g}$ orbitals accompanied by the charge ordering. The orbital ordering and the associated anisotropic transfer integrals leading to the interference of various hopping processes play a crucial role in the electronic structure of the $\mathrm{CO}-\mathrm{OO}$ phase. The direction of orbital stripe (or orbital chain) is closely related to that of the optical anisotropy. The different optical gap value revealed in our spectra is expected to lead to the high anisotropy in dc conductivity. Applying an external electric field might change the direction of the orbital stripe and the corresponding direction of optical anisotropy. In this sense the orbital stripe might work as a parameter of a sort of optical polarization-related device.

Finally we discuss the correlation between the anisotropy and the magnetic correlation by tracing their $T$-dependent evolutions. To get some insight into the anisotropic feature in $\sigma(\omega)$ below $2.5 \mathrm{eV}$, we estimated the effective spectral weight by integration of $\sigma(\omega)$, $N_{\text {eff }}\left(\omega_{c}\right)=\int_{0}^{\omega_{c}} d \omega^{\prime} \sigma\left(\omega^{\prime}\right)$, and found that $N_{\text {eff }}\left(\omega_{c}=2.5\right.$ $\mathrm{eV})$ are nearly identical in both axes. This indicates that the optical anisotropy observed in the CO-OO state is associated with the distribution of the local spectral weight below $2.5 \mathrm{eV}$. To clarify this, we display the differential conductivity spectra $\Delta \sigma(\omega)=\sigma_{a^{\prime}}(\omega)-\sigma_{b^{\prime}}(\omega)$ at various $T$ in Fig. $3(\mathrm{c})$. While the $\Delta \sigma(\omega)$ above $T_{C O-O O}$ exhibits quite a small spectral weight with negligible $T$ dependence, the distinct two peaks with opposite signs, which originate from the accumulation of lower-energy spectral weight along the $a^{\prime}$ axis, develop significantly below $T_{C O-O O}$. We also estimated the differential effective spectral weight $\Delta N_{\text {eff }}=\int_{0}^{\omega_{c}} d \omega^{\prime} \Delta \sigma\left(\omega^{\prime}\right)$ with $\omega_{c}=1.3$ $\mathrm{eV}$, where the $\sigma(\omega)$ in both axes coincide at the lowest $T$. As shown in the inset of Fig. 4(c), $\Delta N_{\text {eff }}$ exhibits a sizable value with a steep increase below $T_{C O-O O}$, and then appears to be rather saturated at lower $T$, not exhibiting a distinct change near $T_{N}$. From this it appears that the three-dimensional (3D) long-range spin ordering does not affect the optical anisotropy significantly. When the orbital ordering occurs, the short-range magnetic correlation could be presumably formed along the zigzag chain even above $T_{N}$, which have been identified by the neutron diffraction measurement for some COOO manganites [3, 6, 19]. No significant change of the anisotropy near $T_{N}$ implies that this ferromagnetic fluctuation along the chain could be effective for the formation of the quasi-1D electron hopping, irrespective of the onset of 3D long-range magnetic order. This may be indicative of the predominant role of the orbital ordering in the magnetic correlation.

In summary, we have presented the experimental and theoretical studies on the optical spectra in the charge/orbital ordered plane with polarization dependence. The in-plane anisotropy is attributed to the peculiar orbital arrangement accompanied by the magnetic correlation.

We thank D. I. Khomskii, Y. Okimoto, M. Uchida, R. Mathieu, X. Z. Yu, and Y. Motome for useful discussions.
[1] M. Imada, A. Fujimori, and Y. Tokura, Rev. Mod. Phys. 70, 1039 (1998).

[2] J.B. Goodenough, Phys. Rev. 100, 564 (1955).

[3] B.J. Sternlieb, J.P. Hill, U.C. Wildgruber, G.M. Luke, B. Nachumi, Y. Moritomo, and Y. Tokura, Phys. Rev. Lett. 76, 2169 (1996).

[4] Y. Murakami, H. Kawada, H. Kawata, M. Tanaka, T. Arima, Y. Moritomo, and Y. Tokura, Phys, Rev. Lett. 80, 1932 (1998).

[5] D.V. Efremov, J. van den Brink, and D.I. Khomski, Nature materials 3, 853 (2004).

[6] S. Larochelle, A. Mehta, L. Lu, P.K. Mang, O.P. Vajk, N. Kaneko, J.W. Lynn, L. Zhou, and M. Greven, Phys. Rev. B 71, 024435 (2005).

[7] Y. Tokura and N. Nagaosa, Science 288, 462 (2000).

[8] K. Tobe, T. Kimura, and Y. Tokura, Phys. Rev. B 69, 014407 (2004).

[9] W.A. Harrison, Electronic Structure and the Properties of Solids (Dover, New York, 1989).

[10] J. Bala, P. Horsch, and Frank Mack, Phys. Rev. B 69, 094415 (2004).
[11] T. Ishikawa, K.Ookura, and Y. Tokura, Phy. Rev. B 59, 8367 (1999).

[12] Y. Tomioka and Y. Tokura, Phys. Rev. B 70, 014432 (2004).

[13] According to the recent x-ray scattering experiment, the $T_{N}$ for the $\operatorname{Pr}_{1 / 2} \mathrm{Ca}_{3 / 2} \mathrm{MnO}_{4}$ compound with the nearly same $T_{C O-O O}$ is found to be $125 \mathrm{~K}$.

[14] Y. Okimoto, Y. Tomioka, Y. Onose, Y. Otsuka, and Y. Tokura, Phys. Rev. B 57, R9377 (1998).

[15] J.H. Jung, J.S. Ahn, Jaejun Yu, T.W. Noh, Jinhyoung Lee, Y. Moritomo, I. Solovyev, and K. Terakura, Phys. Rev. B 61, 6902 (2000).

[16] M. Cuoco, C. Noce, and A.M. Oles, Phys. Rev. B 66, 094427 (2002).

[17] J. Bala and P. Horsch, Phys. Rev. B 72, 012404 (2005).

[18] K. Yamamoto, T. Kimura, T. Ishikawa, T. Katsufuji, and Y. Tokura, Phys. Rev. B 61, 14706 (2000).

[19] F. Ye, J.A. Fernandez-Baca, Pengcheng Dai, J.W. Lynn, H. Kawano-Furukawa, H. Yoshizawa, Y. Tomioka, and Y. Tokura, Phys. Rev. B 72, 212404 (2005). 